\title{
Die Auswirkung der Forderungsabtretung oder der Schuldübernahme auf die Konventionalstrafe
}

\section{The Effect of the Transfer of Receivables or Assumption of Debt to the Penalty Clause}

\author{
Alacağın Devri veya Borcun Üstlenilmesinin Cezaî Şarta Etkisi
}

\section{Irem Yayvak Namli' 1 [}

\begin{abstract}
Zusammenfassung
Obwohl die Konventionalstrafe in vielen Konventionen in der Praxis enthalten ist, enthält sie viele kontroverse Fragen, die sowohl in der Lehre als auch in den Gerichtsentscheidungen noch zu klären sind. Eines dieser Probleme ist das Schicksal der Konventionalstrafe im Falle der Forderungsabtretung oder Schuldübernahme. In dieser Studie wird zunächst das Prinzip der Akzessorietät der Konventionalstrafe erörtert und anschließend die in der türkischen, schweizerischen und deutschen Lehre vertretenen Meinungen einbezogen. Abschließend möchten wir darauf hinweisen, dass die akzessorische Natur der Konventionalstrafe die kontroversen Themen in den Mittelpunkt stellt.
\end{abstract}

\section{Schlüsselwörter}

Konventionalstrafe, Forderungsabtretung, Schuldübernahme, Akzessorietät, Hauptschuld

\begin{abstract}
Although the penalty clause is included in many contracts in practice today, it contains several controversial issues that are still waiting to be resolved both in the doctrine and judicial decisions. One of these issues is the consequence of the penalty clause in the event of the transfer of the receivables or in case the debt is assumed. In our study, first, the accessory nature of the penalty clause will be discussed. Then, the opinions put forward on the subject in the Turkish, Swiss and German doctrines will be included. And finally, we will state our opinion focusing on the accessory nature of the penalty clause on the controversial issues.

Keywords

Penalty clause, Transfer of receivables, Assumption of debt, Accessory obligation, Principal debt

Öz

Cezai şart, uygulamada birçok sözleşmede yer almakla birlikte, gerek doktrinde gerekse yargı kararlarında halen çözümlenmeyi bekleyen birçok tartışmalı konu içermektedir. Bu konulardan biri ise alacağın devri veya borcun üstlenilmesi halinde cezai şartın akıbetidir. Çalışmamızda öncelikle cezai şartın fer'i niteliği ele alınacak olup, daha sonra Türk, İsviçre ve Alman doktrininde konu hakkında ileri sürülen görüşlere yer verilecektir. En son olarak ise tartışmalı hususlarda cezai şartın fer'i niteliğinin odak alındığı kanaatimiz belirtilecektir.
\end{abstract}

\section{Anahtar Kelimeler}

Cezai şart, Alacağın devri, Borcun üstlenilmesi, Fer'i borç, Asıl borç

\footnotetext{
1 Corresponding Author: Irem Yayvak Namlı, (Res. Asst. Dr.) Istanbul University, Faculty of Law, Department of Labor And Social Security Law, Istanbul, Turkey. E-mail: irem.yayvak@istanbul.edu.tr ORCID: 0000-0003-1669-6826

To cite this article: Yayvak Namli, Irem: “Die Auswirkung Der Forderungsabtretung Oder Der Schuldübernahme Auf Die Konventionalstrafe”, Annales de la Faculté de Droit d’Istanbul, 67, 2018, 109-124. https://doi.org/10.26650/annales.2018.67.0007 


\section{Extended Summary}

Although the penalty clause is included in many contracts in practice today, it contains several controversial issues that are still waiting to be resolved both in the doctrine and in judicial decisions. One of these issues is the consequence of the penalty clause in the event of the transfer of receivables or in case the debt is assumed.

The penalty clause is a provision requiring financial compensation, which is promised to be paid to the other party in the event of the contract is being breached, either partially or fully. In Turkish Law, the penalty clause is regulated in Article 179 and in the following sections of the Turkish Code of Obligations number 6098. The penalty clause appears as a debt in connection with the principal debt. The emergence, continuity and termination of the penalty clause depends on the existence of the principal debt. The transfer of the receivable is regulated between Articles 183 and 194 of the Turkish Code of Obligations number 6098. Because of its accessory nature the penalty clause has significant consequences. One of these is the transfer of authority to demand a penalty clause of the new owner of the receivable in the event of the transfer of the said receivable. The Swiss and German doctrines have for many years placed an emphasis on the transfer of the receivable within the framework of the penalty clause. In this context, however, there are no obvious regulations in Turkish Law. Thus, many different views have arisen on the matter. The focus is set on whether the penalty clause can be transferred independently of the principal receivable. Moreover, many different opinions have also emerged about which creditor has the right to demand a penalty clause. The issue is generally handled depending on whether the penalty clause is due. Our present study focuses specifically on this latter detail.

In the event of the transfer of the principal receivable, the penalty clause which is not yet due will also be transferred to the transferee together with the principal receivable. The matter of transferring the penalty clause to a third party independently of the principal receivable before it is due is controversial. After the penalty clause is due, the principal-accessory debt relationship with the principal debt disappears. In this case, since the penalty clause turns into an independent receivable, the penalty clause does not have to pass onto the transferee when the principal receivable is transferred. In the event of the transfer of the receivable, a distinction should be made taking into account the general principles regarding whether the penalty clause can be transferred independently before or after it is due. Therefore, before the penalty clause is due, an interpretation should be made by paying attention to the fact that it has the accessory nature. In our study, our opinion on the controversial issues is explained in detail within the boundaries of this interpretation.

Assuming of debt is regulated in Article 195 and the following sections of the 
Turkish Code of Obligations number 6098. If the debt is assumed, the accessory nature of the penalty clause comes to the fore regarding the status of the penalty clause. According to Article 198/1 of the TBK, the rights of the creditor other than those related to the personality of the debtor will be reserved even if the debtor has changed. This conclusion does not raise any doubt that the person who assumes the debt will be liable if the penalty clause is due after the debt is assumed. An important issue to address in terms of assuming the debt is whether or not it is possible for a third-party to assume the penalty clause, which has not been due yet, independently of the principal debt. Also, an interpretation should be made by paying attention to the accessory nature of the penalty clause in the controversial areas previously mentioned about assuming the debt. Before the penalty clause is due, it is not possible to independently assume this due to the accessory nature of the penalty clause. On the other hand, the penalty clause continues its existence as an independent debt after it is due. In this case, it is possible to assume it separately from the principal debt. In our study, an attempt was made to find a solution to the controversial issues on assuming debt by taking the German and Swiss Laws into account.

It should be noted that an interpretation should be made without forgetting that the penalty clause is an accessory debt if the receivable is transferred or the debt is assumed. When this method is pursued, it will be possible to obtain consistent results in terms of the effect of the transferred receivable or assumed debt on both the penalty clause which is due and the penalty clause which has not been due yet.

In our study, the accessory nature of the penalty clause will first be discussed. Then, the opinions put forward on the subject in the Turkish, Swiss and German doctrines will be included. And finally, we will state our opinion focusing on the accessory nature of the penalty clause on the controversial issues. 


\section{Die Auswirkung der Forderungsabtretung oder der Schuldübernahme auf die Konventionalstrafe}

\section{Einführung}

Heutzutage ist es üblich, dass viele Konventionen die Konventionalstrafe beinhalten ${ }^{1}$. Die Konventionalstrafe ist eine Handlung von wirtschaftlichem Wert, von der versprochen wird, dass sie an den Vertragspartner gezahlt wird, falls der Vertrag überhaupt nicht oder nicht richtig erfüllt wird ${ }^{2}$. Die Konventionalstrafe im türkischen Recht ist ab Artikel 179 des türkischen Obligationenrechts Nr. 6098 aufgeführt worden. Durch die Bestimmung der Konventionalstrafe soll die vertragsgemäße Erfüllung der Forderung gewährleistet werden. Darüber hinaus gehört es zu den Zielen der Konventionalstrafe, Druck für die Schuldentilgung auszuüben und die gesetzliche Gläubigerstellung zu stärken

Die Konventionalstrafe ist eine Handlung, die vom Bestand der Hauptverpflichtung abhängt. Dieses Abhängigkeitsverhältnis wird in der Lehre mit dem Begriff der „Akzessorietät“ umschrieben. Die Tatsache, dass die Konventionalstrafe akzessorisch ist, hat wichtige rechtliche Konsequenzen in Bezug auf die Entstehung, Form, Beendigung der Schulden und viele andere Fragen. Hierbei ist eine der Fragen, bei denen die Akzessorietät von Bedeutung ist, die Auswirkung der Forderungsabtretung oder der Schuldübernahme auf die Konventionalstrafe. In diesem Zusammenhang hat die Forderungsabtretung oder die Schuldübernahme vor oder nach dem Fälligkeitsdatum der Konventionalstrafe unterschiedliche rechtliche Konsequenzen. In dieser Studie werden die rechtlichen Konsequenzen der Forderungsabtretung oder der Schuldübernahme im Hinblick auf die Konventionalstrafe untersucht.

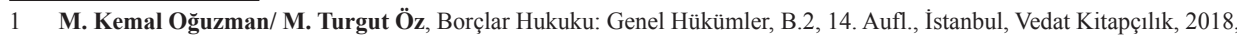
s.534.

2 Selahattin Sulhi Tekinay/ Sermet Akman/ Haluk Burcuoğlu/ Atilla Altop, Tekinay Borçlar Hukuku: Genel Hükümler, 7. Aufl., İstanbul, Filiz Kitabevi, 1993, s.341; Necip Bilge, "Cezaî Şart”, Ahmet Esat Arsebük’ün Aziz Hatırasına Armağan, Ankara, Güzel İstanbul Matbaası, 1958, s.39; Kenan Tunçomağ, Türk Hukukunda Cezai Şart, İstanbul, Baha Matbaası, 1963, s.6; Fikret Eren, Borçlar Hukuku: Genel Hükümler, 23. Aufl., Ankara, Yetkin Yayınları, 2018, s.1209; Oğuzman/ Öz, s.534; Haluk N. Nomer, Borçlar Hukuku: Genel Hükümler, 16. Aufl., İstanbul, Beta, 2018, Rn.235.1; Ferit Hakkı Saymen/ Halid Kemal Elbir, Borçlar Hukuku Dersleri: Umumi Hükümler, B.1, İstanbul, İsmail Akgün Matbaası, 1958, s.553.

3 Manfred Löwisch/ Rainer Jagmann/ Volker Rieble, J. von Staudingers Kommentar zum Bürgerlichen Gesetzbuch: Buch 2: Recht der Schuldverhältnisse: §§ 328-345 (Vertrag zugunsten Dritter, Draufgabe, Vertragsstrafe), Berlin, Sellierde Gruyter, 2015, Vor Art 339, N.16; Rolf Stürner, Jauernig Bürgerliches Gesetzbuch: mit Rom I, Rom II, Rom III-VO, EG-UntVO/HUntProt und EuErbVO, Kommentar, 16. Aufl., München, C.H.Beck, 2015, Art 339, N.3; Hanns Prütting/ Gerhard Wegen/ Gerd Weinreich, Bürgerliches Gesetzbuch, Kommentar, 11. Aufl., Köln, Luchterhand Verlag, 2016, Vor Art 339, N.1; Walter Erman, Bürgerliches Gesetzbuch: Handkommentar, 14. Aufl., § 339-345, Köln, Verlag Dr. Otto Schmidt, 2014, Vor Art 339-345, Rn.1; Reinhard Richardi/ Otfried Wlotzke/ Hellmut Wissmann/ Hartmut Oetker, Münchener Handbuch zum Arbeitsrecht: Individualarbeitsrecht, B. I, 3. Aufl., München, Verlag C.H. Beck, 2009, §39, Rn.48; Alfred Söllner, "Vertragsstrafen im Arbeitsrecht", AuR, 1981, s.98; Jan-Malte Niemann, "Vertragsbruch: Strafabreden in Formularbeitsverträgen", RdA, 2013, Heft 2, s.93; Hermann H. Haas/ Michael Fuhlrott, "Ein Plädoyer für mehr Flexibilität bei Vertragsstrafen", NZA-RR, 2010/1, s.1. Siehe auch, Tekinay/ Akman/ Burcuoğlu/ Altop, s.342. Siehe auch, BGH, 20.01.2000, v11 zr 46/98, NJW 2000, 2106. 


\section{Die akzessorische Natur der Konventionalstrafe}

Die Konventionalstrafe dient dazu, die ordnungsgemäße Erfüllung der Hauptschuld sicherzustellen. Mit anderen Worten, es wird als Garantie für die Hauptschuld entschieden. Infolgedessen hängt die Konventionalstrafe von Bestand und Gültigkeit der Hauptschuld ab, aber nicht umgekehrt ${ }^{4}$. Wenn also keine Hauptschulden vorliegen, gibt es keine Konventionalstrafe ${ }^{5}$. Daher ist für den Begriff der Konventionalstrafe dieser akzessorische Zusammenhang wesentlich ${ }^{6}$.

Das Verhältnis zwischen der Konventionalstrafe und der Hauptschuld besteht bis zum Fälligkeitsdatum der Konventionalstrafe fort. Nach dem Fälligkeitsdatum wird die Strafe eine eigenständige Schuld. Daher hat der Verfall der Hauptschuld aus irgendeinem Grund nach Fälligkeit keine Auswirkungen auf die Konventionalstrafe ${ }^{7}$. Somit kann die Konventionalstrafe unabhängig vom Schicksal der Hauptschuld geltend gemacht werden ${ }^{8}$.

Die Entstehung, Fortbestand und Verfall der akzessorischen Nebenschuld hängt jedoch vom Bestehen der Hauptschuld $a b^{9}$. Die Beziehung zwischen der Konventionalstrafe und der Hauptschuld ist tatsächlich einseitig. Also, die Konventionalstrafe richtet sich nach der Hauptschuld; die Hauptschuld hängt jedoch nicht vom Vorliegen der Konventionalstrafe ab. Dieses Resultat wird in dem Türkischen Obligationenrecht Art.182/2 deutlich erläutert. Im schweizerischen Obligationenrecht wird es nicht einmal erwähnt. Der Verfall der Konventionalstrafe aus irgendeinem Grund keine Auswirkungen auf die Gültigkeit der Hauptschuld ${ }^{10}$.

4 Eugen Bucher, Schweizerisches Obligationenrecht: Allgemeiner Teil ohne Deliktsrecht, 2. Aufl., Zürich, Schulthess Polygraphischer Verlag, 1988, s.523; Rudolf M. Reck, Lohnrückbehalt, Kaution und Konventionalstrafe im schweizerischen Arbeitsrecht, Zürich, ADAG Administration \& Druck AG, 1983, s.99 und 110; Andreas Von Tuhr/ Arnold Escher, Allgemeiner Teil des Schweizerischen Obligationenrechts, B. II, 3. Aufl., Zürich, Schulthess Polygraphischer Verlag AG, 1974, s.278; Roland Bentele, Die Konventionalstrafe nach Art. 160-163 OR, Freiburg-Schweiz, Paulusdruckerei, 1994, s.31; Jauernig, Art 339, N.2.

5 Franz Jürgen Säcker/ Roland Rixecker/ Hartmut Oetker/ Bettina Limperg, Münchener Kommentar zum Bürgerlichen Gesetzbuch, B. II: Schuldrecht-Allgemeiner Teil, 7. Aufl., München, C. H. Beck, 2016, Art 339, Rn.14; Von Tuhr/ Escher, s.278; Walter Schoch, Begriff, Anwendung und Sicherung der Konventionalstrafe nach schweizerischem Recht, Bern, Stämpfli \& Cie., 1935, s.16; Bucher, s.523; Prütting/ Wegen/ Weinreich, Vor Art 339, N.2; MüArbR/Reichold, §39, Rn.48; Reck, s.99; Peter Gauch/ Walter R. Schluep/ Jörg Schmid/ Heinz Rey, Schweizerisches Obligationenrecht Allgemeiner Teil: ohne ausservertragliches Haftpflichtrecht, B. II, 7.Aufl., Zürich, Schulthess Polygrapischer Verlag, 1998, Rn.3959; Bentele, s.31; Otto Palandt, Bürgerliches Gesetzbuch mit Nebengesetzen, B. VII, 76. Aufl., München, C.H. Beck Verlag, 2017, s.570; Niemann, s.92; Alfred Koller, Schweizerisches Obligationenrecht Allgemeiner Teil: Handbuch des allgemeinen Schuldrechts ohne Deliktsrecht, 3. Aufl., Bern, Stämpfli Verlag AG, 2009, § 81, Rn.4; Saymen/ Elbir, s.554; Bilge, s.67; Tunçomağ, Cezaî Şart, s.15; Oğuzman/ Öz, s.541; Tekinay/ Akman/ Burcuoğlu/ Altop, s.342-343; Nomer, Rn.237; Ayça Akkayan Yıldırım, "Cezai Şartın İşlevi Türk ve Amerikan Hukukları Açısından Karşılaştırmalı Bir Değerlendirme”, IÜHFM, B. 61, N.1-2, 2003, s.366-367.

6 Tunçomağ, Cezaî Şart, s.15; Oğuzman/ Öz, s.541; Tekinay/ Akman/ Burcuoğlu/ Altop, s.342; MüKoBGB/ Gottwald, Art 339, Rn.14; Jauernig, Art 339, N.17; Haas/ Fuhlrott, s.1; Niemann, s.92.

7 Von Tuhr/ Escher, s.279; Reck, s.100; Dimitri Santoro, Die Konventionalstrafe im Arbeitsvertrag, Bern, Stämpfli Verlag AG, 2001, s.10 Tunçomağ, Cezaî Şart, s.16; Bilge, s.74; Eren, s.1211; Akkayan Yıldırım, s.367.

8 Tunçomağ, Cezaî Şart, s.16; Eren, s.1211; Von Tuhr/ Escher, s.279; Reck, s.100; Santoro, s.10.

9 Tunçomağ, Cezaî Şart, s.15; Oğuzman/ Öz, s.541; Tekinay/ Akman/ Burcuoğlu/ Altop, s.342-343; Nomer, Rn.237.

10 Von Tuhr/ Escher, s.279; Reck, s.100; Santoro, s.7; Saymen/ Elbir, s.554; Oğuzman/ Öz, s.543-544; Nomer, Rn.237.5. 


\section{Konventionalstrafe im Falle der Forderungsabtretung}

Die Forderungsabtretung basiert auf das türkische Obligationenrecht Nr. 6098 Art.183-194. Die Forderungsabtretung ist die Abtretung der Forderungen aus einem Schuldverhältnis durch den zwischen dem Schuldner und dem Gläubiger abgeschlossenen Vertrag entstanden worden ist ${ }^{11}$. Eine wichtige Folge der Tatsache, dass es sich bei der Konventionalstrafe um eine akzessorische Natur handelt, ist die Abtretung der Befugnis, die Konventionalstrafe zu beantragen, wenn die Forderung an den Dritten abgetreten wird ${ }^{12}$.

Normalerweise hat jeder, der eine Schuldenbeziehung eingeht, den Titel eines Gläubigers und Schuldners. In den Artikeln 183 des türkischen Obligationenrechts und ihrer Fortführung ist jedoch festgelegt, dass die Forderungen ohne Einwilligung des Schuldners an einen andern abgetreten werden können. Solange das Gesetz, die Vereinbarung oder die Natur des Rechtsverhältnisses diese Abtretung nicht entgegenstehen. Die Forderungsabtretung bedarf zu ihrer Gültigkeit der schriftlichen Form $^{13}$.

In der schweizerischen und deutschen Lehre wird seit vielen Jahren die Frage der Abtretung im Rahmen der Konventionalstrafe behandelt. Insbesondere zu den umstrittenen Fragen, ob die Konventionalstrafe unabhängig von der Hauptforderung abtreten werden kann und welcher Gläubiger zur Forderung der Konventionalstrafe berechtigt ist, haben sich viele unterschiedliche Meinungen ergeben. Das Problem wird normalerweise behandelt, je nachdem, ob die Konventionalstrafe fällig ist oder nicht. In Bezug auf die Systematik der Studie wird das Thema durch diese Unterscheidung untersucht.

\section{A. Die Auswirkung der Abtretung von Hauptforderungen auf die nicht fällige Konventionalstrafe}

Im Falle der Abtretung der Hauptforderung wird die noch nicht fällige Konventionalstrafe zusammen mit der Hauptforderung auch an den Erwerber

11 Von Tuhr/ Escher, s.329, Max Gmür, Kommentar zum Schweizerischen Zivilgesetzbuch: Obligationenrecht Art 68-183 (Becker), B. VI, Bern, Verlag von Stämpfli Cie., 1917, OR Art 164, N.1; Bucher, s.536; Theo Guhl/ Alfred Koller/ Anton Schnyder/ Jean Nicolas Druey, Das Schweizerische Obligationenrecht: mit Einschluss des Handels- und Wertpapierrechts, 9. Aufl., Zürich, Schulthess, 2000, §34, Rn.1; Tekinay/ Akman/ Burcuoğlu/ Altop, s.240; Kenan Tunçomağ, Türk Borçlar Hukuku, Genel Hükümler, B. I, 6. Aufl., İstanbul, Sermet Matbaası, 1976, s.644; Tolunay Ozanemre Yayla, Alacağın Devri İşleminin Geçerliliği ve Sebeple Olan İlişkisi (İllîliği), Ankara, Turhan Kitabevi, 2019, s.42.

12 Hugo Oser/ Wilhelm Schönenberger, Zürcher Kommentar zum Schweizerischen Zivilgesetzbuch, B. V: Erster Halbband, Obligationenrecht, Art 1-183 OR, 2. Aufl., Zürich, Schulthess, 1929, Vor. zu Art. 160-163 OR, Rn.18; Tunçomağ, Cezaî Şart, s.15; MüKoBGB/ Gottwald, Art 339, Rn.15; Schoch, s.48; Palandt/ Grüneberg, s.572; Prütting/ Wegen/ Weinreich, Vor Art 339, N.2; Erman/ Schaub, Art 339, Rn.4; Oğuzman/ Öz, s.561; Bilge, s.74; Tekinay/ Akman/ Burcuoğlu/ Altop, s.250-251.

13 Bentele, s.100-101; Oser/ Schönenberger, Art 164 OR, Rn.8; Schoch, s.50; Von Tuhr/ Escher, s.329; Oğuzman/Öz, s.562; Tunçomağ, Türk Borçlar Hukuku, s.1074 usw.; Tekinay/ Akman/ Burcuoğlu/ Altop, s.240; Eren, s.1252 usw.; Kemal Dayınlarlı, Borçlar Kanununa Göre Alacağın Temliki, 4. Aufl., Ankara, Dayınlarlı Hukuk Yayınları, 2010, s.76 usw., s.62 usw.; Ozanemre Yayla, s.225 usw. 
weitergegeben. Tatsächlich wird diese Angelegenheit im türkischen Obligationenrecht Art.189 mit folgenden Worten angegeben "Vorzugsrechte und Nebenrechte gehen mit der Forderung über, mit Ausnahme derer, die untrennbar mit der Person des Abtretenden verknüpft sind. " Es wird nicht einmal angestrebt, dass sich die Parteien auf diese Angelegenheit einigen ${ }^{14}$.

Es ist fraglich, ob es unabhängig von der Hauptforderung an einen Dritten abtreten werden kann oder nicht, bevor die Konventionalstrafe fällig wird. Nach deutscher Rechtsprechung kann es in Ausnahmefällen möglich sein, die noch nicht fällige Konventionalstrafe unabhängig von der Hauptforderung abzutreten ${ }^{15}$. Trotz der Forderungsabtretung nach dieser Meinung bleibt die Befugnis beim Gläubiger der Hauptforderung, die Konventionalstrafe aufgrund der Akzessorietät zu verlangen ${ }^{16}$. Im türkischen Recht gibt es auch Autoren, die die Meinung vertreten, dass noch nicht fällige Forderungen als erwartete Rechte abtreten werden können, wenn die Forderung festgestellt oder identifizierbar ist. In diesem Fall entstehen zukünftige Forderungen direkt aus dem Vermögen des Erwerbers ${ }^{17}$.

\section{B. Die Auswirkung der Abtretung der Hauptforderungen auf die fällige Konventionalstrafe}

Nach Fälligkeit der Konventionalstrafe wird die Beziehung zwischen den Nebenrechten und der Hauptforderung aufgehoben ${ }^{18}$. In diesem Fall muss die Konventionalstrafe bei der Abtretung der Hauptforderung nicht auf den Erwerber übergehen, da die Konventionalstrafe zu einer selbstständigen Forderung geworden ist. Es kann jedoch von den Vertragsparteien entschieden werden, ob die Konventionalstrafe zusammen mit der Hauptforderung an den Erwerber weitergegeben wird. Andernfalls wird die Konventionalstrafe nicht auf den Erwerber abgetreten und verbleibt in der Vermögen des Abtreters ${ }^{19}$. In der Lehre ist jedoch noch umstritten, ob die fällige Konventionalstrafe unabhängig von der Hauptforderung abtreten werden kann.

Die gesetzlichen Bestimmungen zu diesem Thema haben keine deutliche Regelung. Ein schweizerischer Autor besagt, dass die Konventionalstrafe unabhängig von der Hauptschuld weder vor noch nach dem Fälligkeitsdatum abtreten werden kann. Denn

14 Kurt Schellhammer, Schuldrecht nach Anspruchsgrundlagen: samt BGB Allgemeiner Teil, 9. Aufl., Heidelberg, C.F.Müller, 2014, Rn.1487; Bentele, s.32; Schoch, s.49; Becker, Art 160 OR, N.25; Gauch/ Schluep/ Rey, Rn.4060; Oğuzman/ Öz, s.581 usw.; Eren, s.1268; Bilge, s.74; Dayındarlı, s.196; Köksal Kocaağa, Ceza Koşulu (Sözleşme Cezas1), 2. Aufl., Ankara, Yetkin Yayınları, 2018, s.159.

15 MüKoBGB/ Gottwald, Art 339, Rn.15.

16 MüKoBGB/ Gottwald, Art 339, Rn.15. Siehe auch, Palandt/ Grüneberg, s.571.

17 Tunçomağ, Türk Borçlar Hukuku, s.1082. Siehe auch, Tekinay/ Akman/ Burcuoğlu/ Altop, s.248-249; Dayınlarlı, 2010, s.163; Von Tuhr/ Escher, s.349; Becker, Art 164, N.16.

18 Von Tuhr/ Escher, s.279; Reck, s.100; Tunçomă̆, Cezaî Şart, s.16; Eren, s.1211.

19 Von Tuhr/ Escher, s.356; Bilge, s.75; Kocaağa, s.160. 
die Konventionalstrafe hängt immer noch von der Hauptschuld ab. Andernfalls kann der Zweck der Sicherung der Forderung durch eine Konventionalstrafe nicht erreicht werden. Darüber hinaus wird gezeigt, dass es nicht angemessen ist, dass die dritte Person, die nicht einmal das Recht hat, die Erfüllung der Hauptschuld zu verlangen, Konventionalstrafe verlangen kann ${ }^{20}$.

In der Doktrin wird auch die Auffassung vertreten, dass die Abtretung der Konventionalstrafe unabhängig von der Hauptschuld, die vor der Abtretung der Schuld fällig ist oder nicht, keinen Nachteil anrichtet ${ }^{21}$. Nach dieser Auffassung bewirkt die Abtretung der Konventionalstrafe keinen Unterschied in der Situation des Schuldners. In der Tat gibt es kein negatives Ergebnis für den Schuldner, um die Konventionalstrafe für den Abtretende oder Erwerber zu erfüllen. Nach einer ähnlichen Auffassung kann die Konventionalstrafe eigenständig abgetreten werden, da das Recht, eine Forderung geltend zu machen, nach Fälligkeit der Konventionalstrafe unabhängig wird ${ }^{22}$.

Eine andere Meinung zu diesem Thema unterscheidet ohne Angabe von Gründen zwischen den Arten der Konventionalstrafe. Dementsprechend kann die zur Ausführung kumulative Konventionalstrafe nach Fälligkeit unabhängig abgetreten werden. Andererseits kann die alternative Konventionalstrafe nach ihrer Fälligkeit nicht getrennt von der Hauptschuld abgetreten werden ${ }^{23}$. Der Grund für diese Ansicht ist wahrscheinlich der Wunsch, Streitigkeiten über das Wahlrecht der Gläubiger in der alternativen Konventionalstrafe zu vermeiden.

Im Hinblick auf diese Diskussion gibt es eine andere Meinung, die "Recht" und "Forderungsanspruch" unterscheidet. Nach dieser Auffassung sind das Recht auf eine Konventionalstrafe und der Anspruch auf das Recht auf eine Konventionalstrafe unterschiedliche Begriffe ${ }^{24}$. Tatsächlich ist das Recht auf eine Konventionalstrafe ein weiter gefasster Begriff, da er den Forderungsanspruch einschließt, eine Konventionalstrafe zu verlangen. Der Gläubiger kann sein Recht, eine Konventionalstrafe zu verlangen, auf einen Dritten abtreten. Der Forderungsanspruch verbleibt nicht unbedingt bei der Person, die das Recht auf eine Konventionalstrafe hat. Das Recht auf eine Konventionalstrafe kann jedoch nicht auf den Dritten abgetreten werden und verbleibt beim Gläubiger selbst; weil dieses akzessorische

20 Josef Kohler, Lehrbuch des bürgerlichen Rechts, Berlin, Heymann, 1904, 143, §50.

21 Paul Oertmann, Kommentar zum Bürgerlichen Gesetzbuche und seinen Nebengesetzen: Das Recht der Schuldverhältnisse, 2. Aufl., Berlin, Heymann, 1906, 270, §399; Bentele, s.100; Becker, Art 164, N.15; Oser/Schönenberger, Art 164 OR, Rn.4. Vgl. Tunçomağ, Cezaî Şart, s.88.

22 Max Stahel, Die Konventionalstrafe mit spezieller Berücksichtigung des Schweizerischen Obligationenrechts, Zürich 1898, s.79-80; MüKoBGB/ Gottwald, Art 339, Rn.15.

23 Manfred Löwisch/ Volker Rieble/ Jan Busche/ Dirk Looschelders, J. von Staudingers Kommentar zum Bürgerlichen Gesetzbuch, Buch 2 - Recht der Schuldverhältnisse: $\S$ 397-432 (Erlass, Abtretung, Schuldübernahme, Mehrheit von Schuldnern und Gläubigern), Berlin, Sellier-de Gruyter, 2017, Art 401, Rn.281.

24 Schoch, s.50-51; Tekinay/ Akman/ Burcuoğlu/ Altop, s.249-250. 
Recht nicht vom Hauptrecht getrennt werden kann. Nach deutschem Recht wird anerkannt, während dem Dritten die Befugnis abgetreten werden kann, anstelle der Abtretung der Forderungen die Leistung in seinem Namen zu verlangen ${ }^{25}$. In diesem Fall behält der Gläubiger den Titel des Gläubigers. Der Vertreter dieser Ansicht im türkischen Recht ist $\mathrm{Akyol}^{26}$. Eine andere Ansicht im türkischen Recht kritisiert diese Ansicht jedoch und argumentiert, dass der „Forderungsanspruch“" und das „Recht“" nicht getrennt werden können, da diese eng miteinander verbunden sind ${ }^{27}$.

Ein weiteres wichtiges Problem ergibt sich, wenn die Auffassung vertreten wird, dass die Abtretung der Konventionalstrafe unabhängig von der Hauptschuld erfolgt. Gehört in diesem Fall das Recht, alternative Konventionalstrafe zu verlangen, dem Abtretende oder dem Erwerber? Zunächst ist festzuhalten, dass sich die Parteien im Vertrag eindeutig auf dieses Thema einigen können ${ }^{28}$. Die Tatsache, dass es diesbezüglich im Vertrag keine Regelung gibt, wird in der Doktrin erörtert. Einer Meinung nach wenn die Konventionalstrafe auf den neuen Gläubiger unabhängig von der Hauptforderung abgetreten wird, sollte das Wahlrecht auch auf den Erwerber übergehen. Denn der Erwerber ist bestrebt, mit der Abtretung alle rechtlichen Konsequenzen zu tragen ${ }^{29}$. Andererseits wird in der Doktrin hervorgebracht, dass der Abtretende, der die Hauptforderung hat, in der Regel das Wahlrecht hat ${ }^{30}$.

\section{Unsere Meinung}

Im Rahmen der allgemeinen Grundsätze ist $\mathrm{zu}$ unterscheiden, ob die Konventionalstrafe im Rahmen der Forderungsabtretung selbstständig nach oder vor Fälligkeit abgetreten werden kann.

Erstens kann die Konventionalstrafe vor Fälligkeit nicht selbstständig abgetreten werden, da die Konventionalstrafe akzessorische Natur hat. Infolgedessen übernimmt man mit der die Hauptforderung auch die mit der Forderung verbundene Konventionalstrafe. Daher wäre es angemessener, eine Stellungnahme abzugeben, bevor die Konventionalstrafe fällig wird, ohne deren akzessorischen Charakter zu vernachlässigen.

Zweitens sollte berücksichtigt werden, dass die Abtretung der Konventionalstrafe auch unserer Meinung nach von der Hauptforderung unabhängig ist, nachdem die Konventionalstrafe fällig ist. Sofern im Vertrag nichts anderes vereinbart ist, steht der selbständigen Weitergabe der Konventionalstrafe an den Dritten unabhängig

\footnotetext{
25 Karl Larenz, Allgemeiner Teil des Deutschen Bürgerlichen Recht, 6. Aufl., München, C.H. Beck Verlag, 1983, s.233.

26 Şener Akyol, Alacaklının Verdiği Üçüncü Şahsın İfayı Kendi Adına Talep Yetkisi, İstanbul 1981, s.7 usw.

27 Tekinay/ Akman/ Burcuoğlu/ Altop, s.249-250.

28 Tunçomağ, Cezaî Şart, s.90; Bentele, s.101.

29 Tunçomağ, Cezaî Şart, s.89-90.

30 Becker, Art 160 OR, N.25; Bilge, s.75.
} 
vom Schicksal der Hauptforderung daher nichts im Wege. In diesem Fall ist jedoch vor allem zu erörtern, welcher Gläubiger berechtigt ist, eine Konventionalstrafe zu verlangen, wenn die Konventionalstrafe unabhängig auf einen Dritten abgetreten wird. In diesem Fall sind wir der Ansicht, dass das Wahlrecht zwischen der Erfüllung der Hauptforderung und der Konventionalstrafe nicht voneinander getrennt werden sollte, insbesondere nicht im Hinblick auf die alternative Konventionalstrafe. In diesem Fall führt die getrennte Gewährung des Anspruchs beider Gläubiger zu der Frage, welcher Gläubiger von seinem Wahlrecht Gebrauch gemacht hat, und beseitigt das Bestehen des Wahlrechts, das das wichtigste dem Eigentümer der Hauptforderung eingeräumte Recht ist. Aus diesem Grund ist es die am besten geeignete Lösung, beim Gläubiger zu bleiben, der das Recht hat, die Hauptforderung zu verlangen.

In Bezug auf die alternative Konventionalstrafe betonen wir, dass wir der Meinung sind, dass die Wahl des Gläubigers zwischen der Erfüllung der Hauptforderung und der Konventionalstrafe laut dem türkischen Obligationenrecht Art. 179/1 als "Wahlrecht" zugelassen werden sollte. Das Wahlrecht ist in diesem Zusammenhang ein Gestaltungsrecht und geht in der Regel mit der Abtretung der Forderung auf den Erwerber einher ${ }^{31}$. Nach der Lehre, an der wir uns anschließen, geht das Gestaltungsrecht jedoch nicht auf den Erwerber über, wenn dieser verpflichtet ist, Vertragspartner des entstandenen Vertrags zu sein ${ }^{32}$. Wenn wir uns mit unserem Thema auseinandersetzen, wenn die fällige alternative Konventionalstrafe unabhängig von der Hauptforderung abtreten wird, das Wahlrecht tatsächlich mit der Hauptforderung zusammenhängt, wird der Anspruch auf das Recht auf eine Konventionalstrafe nicht auf den Erwerber übertragen. Dieses Recht verbleibt beim Abtretenden. Wird die Konventionalstrafe auch an eine andere Person abgetreten, der die Konventionalstrafe nicht übernimmt, sollte anerkannt werden, dass das Wahlrecht beim Erwerber liegt, der die Hauptforderung hat.

In diesem Fall, wenn der Gläubiger der Hauptforderung sich für die Erfüllung der Konventionalstrafe entscheidet, sollte die Konventionalstrafe an dem Erwerber erfüllt werden; in der Tat ist er der Inhaber der Konventionalstrafe. Wenn der Inhaber der Hauptforderung hingegen sein Wahlrecht für die Erfüllung der Hauptforderung ausübt, kann der Erwerber die Konventionalstrafe nicht mehr geltend machen. Mit diesem Ergebnis sollte nicht davon ausgegangen werden, dass der Erwerber einen Rechtsverlust erlitten hat. Daher sollte diese Situation im Abtretungsvertrag berücksichtigt werden, und es sollten Vorkehrungen mit den Bestimmungen getroffen werden, um einen Interessenausgleich in der internen Beziehung sicherzustellen.

31 Von Tuhr/ Escher, s.356-357; Oğuzman/ Öz, s.583; Tekinay/Akman/ Burcuoğlu/ Altop, s.251; Eren, s.1268.

32 Von Tuhr/ Escher, s.342-343; Peter Gauch/ Walter R. Schluep/ Jörg Schmid/ Heinz Rey, Schweizerisches Obligationenrecht Allgemeiner Teil: ohne ausservertragliches Haftpflichtrecht, B. I, 7.Aufl., Zürich, Schulthess Polygrapischer Verlag, 1998, Rn.3459 usw.; Oğuzman/ Öz, s.583-584; Tekinay/ Akman/ Burcuoğlu/ Altop, s.251; Eren, s.1268. 


\section{Konventionalstrafe bei der Schuldübernahme}

\section{A. Die Auswirkung der Schuldübernahme auf die Konventionalstrafe}

Die Übernahme der Schuld ist im türkischen Obligationenrecht Nr. 6098 Art.195 aufgeführt. In der Doktrin gibt es eine allgemeine Definition dieses Konzepts für den Fall, dass jemand Schulden übernimmt und zum Schuldner wird, unter der Bedingung, dass die Schuld in einem Schuldverhältnis steht, an dem er zuvor nicht teilgenommen hat ${ }^{33}$. Zur Schuldübernahme ist auch die Zustimmung des Gläubigers erforderlich.

Im Falle einer Schuldübernahme zeigt sich hier in Bezug auf sein Schicksal auch die akzessorische Natur der Konventionalstrafe. In Artikel 198/1 des türkischen Obligationenrechts ist geregelt, dass auch bei einem Schuldnerwechsel die Nebenrechte des Gläubigers, die nicht mit der Person des bisherigen Schuldners untrennbar verknüpft sind, vorbehalten bleiben. Infolge des zwischen dem Gläubiger und dem Übernehmer abgeschlossenen Vertrages gehen dementsprechend die Hauptschuld und folglich die Konventionalstrafe auf den Übernehmer über ${ }^{34}$. Diese Schlussfolgerung lässt keine Zweifel daran aufkommen, dass die Haftung zu vertreten ist, wenn die Konventionalstrafe nach der Schuldübernahme fällig wird. Aus diesem Artikel kann jedoch nicht hervorgeholt werden, von welchem Schuldner die Konventionalstrafe verlangt werden soll, die fällig ist, bevor die Schuld übernommen wurde. Eine Ansicht in der Doktrin besagt, dass in diesem Fall die Konventionalstrafe vom bisherigen Schuldner verlangt werden sollte ${ }^{35}$; in der anderen Ansicht wird besagt, dass der Schuldner angefordert werden soll, der die Schuld übernommen hat ${ }^{36}$. Nach der ersten Ansicht wird die fällige Konventionalstrafe ihren akzessorischen Charakter verlieren. Sofern im Vertrag nicht anders vereinbart ist, geht die Schuld daher nicht auf den Übernehmer über. Nach der zweiten Ansicht werden jedoch sowohl vor als auch nach dem Fälligkeitsdatum die Konventionalstrafe vom Übernehmer verlangt. Denn die gegenteilige Annahme widerspricht dem Zweck und der Qualität des Instituts. Die Parteien können jedoch etwas anderes vereinbaren ${ }^{37}$. Tatsächlich gilt bei der Übernahme der Schuld auch der Übernehmer, der keinen Wille hat, die fällige Konventionalstrafe nicht zu bezahlen, als die Konventionalstrafe übernehmend ${ }^{38}$.

33 Oser/ Schönenberger, Vor. Art 178 OR, Rn.1; Didem Özcan, Borcun Üstlenilmesi, İstanbul, On İki Levha Yayıncılık, 2017, s.6.

34 Oser/ Schönenberger, Vor. Art 178 OR, Rn.3; Becker, Art 178 OR, N.2; Max Keller / Christian Schöbi, Allgemeine Lehren des Vertragsrechts: Das Schweizerische Schuldrecht, B. I, 3. Aufl., Basel/Frankfurt, Helbing \& Lichtenhahn, 1988, s.80; Zafer Kahraman, Karşılaştırmalı Hukukta Borcun Dış Üstlenilmesi (Borcun Nakli), İstanbul, Vedat Kitapçılık, 2013, s.249.

35 Oser/ Schönenberger, Art 178 OR, Rn.3.

36 Von Tuhr/ Escher, s.392; Gauch/ Schluep/ Rey, Rn.3801; Oğuzman/ Öz, s.617-618; Tekinay/ Akman/ Burcuoğlu/ Altop, s.276-277; Eren, s.1279; Kahraman, s.253-254; Kocaağa, s.161.

37 Von Tuhr/ Escher, s.392; Keller/ Schöbi, s.80; Gauch/ Schluep/ Rey, Rn.3801; Oğuzman/ Öz, s.617-618; Tekinay/ Akman/ Burcuoğlu/ Altop, s.276-277; Tunçomağ, Türk Borçlar Hukuku, s.1133; Ferit Hakkı Saymen, Borçlar Hukuku Dersleri, Umumi Hükümler, B. I, İstanbul, Akgün Matbaası, 1950, s.269, Kocaağa, s.161.

Becker, Art 178 OR, N.2; Keller/ Schöbi, s.80; Kahraman, s.254. 
Ein wichtiger Aspekt, der bei der Übernahme der Hauptschuld und der Konventionalstrafe durch den Dritten berücksichtigt werden sollte, ist, ob es dem Dritten möglich ist, die noch nicht fällige Konventionalstrafe unabhängig von der Hauptschuld zu übernehmen. Einer Meinung nach ist es nicht möglich, dass der Schuldner der Konventionalstrafe ein Dritter ist, der nicht der Schuldner der Hauptschuld ist. Dies liegt daran, dass der Schuldner verpflichtet ist, die Schulden an den Gläubiger zu zahlen ${ }^{39}$. Nach gegenteiliger Meinung kann die Konventionalstrafe unabhängig von der Hauptschuld auf den Dritten übertragen werden. Tatsächlich wird davon ausgegangen, dass bedingte und zukünftige Schulden von Dritten in Bezug auf die Konventionalstrafe übernommen werden können ${ }^{40}$.

\section{B. Unsere Meinung}

In Bezug auf die Schuldübernahme sollten wir auch auf die akzessorische Natur der Konventionalstrafe eingehen, die in der Forderungsabtretung erwähnt wird.

Aufgrund der Akzessorietät ist es nicht möglich, die Hauptschuld unabhängig zu übernehmen, bevor die Konventionalstrafe fällig wird. Wenn die Hauptschuld auf den Dritten übertragen wird, ist der Dritte nunmehr an die Konventionalstrafe gebunden. Zum anderen bleibt die Konventionalstrafe nach Fälligkeit als eigenständige Schuld bestehen. In diesem Fall ist es möglich, sie getrennt von der Hauptschuld zu übernehmen. Denn aufgrund der Konventionalstrafe, die durch den Verlust ihrer akzessorische Natur selbständig geworden ist, kann sie nur dann auf den Schuldner übergehen, wenn dies im Vertrag gesondert geregelt ist.

Im letzten Fall, wenn die Hauptschuld nicht übernommen wird und nur die selbstständige Konventionalstrafe übernommen wird, hat der Gläubiger zwei Möglichkeiten. Mit anderen Worten, wenn der Gläubiger die Erfüllung der Hauptforderung wählt, sollte er / sie einen Antrag vom Schuldner dieser Forderung stellen. Wenn der Gläubiger von seinem Recht Gebrauch macht, das Recht auf Zahlung einer Konventionalstrafe zu wählen, wird er die Strafe vom Schuldner dieser Forderung verlangen. Kurz gesagt, jeder Schuldner sollte für seine eigenen Schulden verantwortlich sein.

\section{Fazit}

Im Falle der Forderungsabtretung oder der Schuldübernahme ist das Schicksal der Konventionalstrafe insbesondere im schweizerischen und deutschen Recht seit langem umstritten. Da es im türkischen Recht keine klare Regelung gibt, sind in diesem Zusammenhang viele unterschiedliche Meinungen zu diesem Thema entstanden.

39 Bilge, s.75.

40 Tunçomağ, Cezaî Şart, s.90; Kahraman, s.253. 
Aufgrund der Auslegung, die vorgenommen werden muss, ohne zu vergessen, dass die Konventionalstrafe akzessorische Natur hat, ist es jedoch möglich, hinsichtlich der Auswirkung der Forderungsabtretung oder der Schuldübernahme sowohl auf die fälligen als auch auf die nicht fälligen Konventionalstrafen einheitliche Schlussfolgerungen zu ziehen.

Grant Support: The author received no grant support for this work. 


\section{Abkürzungen/ List of Abbreviations}

$\begin{array}{ll}\text { Art. } & \text { : Artikel } \\ \text { Aufl. } & \text { : Auflage } \\ \text { AuR } & \text { : Arbeit und Recht } \\ \text { B. } & : \text { Band } \\ \text { IÜHFM } & \text { : İstanbul Üniversitesi Hukuk Fakültesi Mecmuas1 } \\ \text { N. } & \text { : Nummer } \\ \text { NZA-RR } & \text { : Rechtsprechungs-Report Arbeitsrecht } \\ \text { OR } & \text { : Obligationenrecht } \\ \text { RdA } & : \text { Recht der Arbeit } \\ \text { Rn. } & : \text { Randnummer } \\ \text { S. } & : \text { Seite } \\ \text { Vgl. } & : \text { Vergleich }\end{array}$

\section{Literaturverzeichnis/Bibliography}

Akkayan Yıldırım, Ayça: "Cezai Şartın İşlevi Türk ve Amerikan Hukukları Açısından Karşılaştırmalı Bir Değerlendirme”, İÜHFM, B. 61, N.1-2, 2003, s.357-414.

Akyol, Şener: Alacaklının Verdiği Üçüncü Şahsın İfayı Kendi Adına Talep Yetkisi, İstanbul 1981.

Bentele, Roland: Die Konventionalstrafe nach Art. 160-163 OR, Freiburg-Schweiz, Paulusdruckerei, 1994.

Bilge, Necip: “Cezaî Şart”, Ahmet Esat Arsebük'ün Aziz Hatırasına Armağan, Ankara, Güzel İstanbul Matbaas1, 1958, s.39-128.

Bucher, Eugen: Schweizerisches Obligationenrecht: Allgemeiner Teil ohne Deliktsrecht, 2. Aufl., Zürich, Schulthess Polygraphischer Verlag, 1988.

Dayınlarlı, Kemal: Borçlar Kanununa Göre Alacağın Temliki, 4. Aufl., Ankara, Dayınlarlı Hukuk Yayınları, 2010.

Eren, Fikret: Borçlar Hukuku: Genel Hükümler, 23. Aufl., Ankara, Yetkin Yayınları, 2018.

Erman, Walter: Bürgerliches Gesetzbuch: Handkommentar, 14. Aufl., § 339-345, Köln, Verlag Dr. Otto Schmidt, 2014.

Gauch, Peter/ Schluep, Walter R./ Schmid, Jörg/ Rey, Heinz: Schweizerisches Obligationenrecht Allgemeiner Teil: ohne ausservertragliches Haftpflichtrecht, B. I, 7.Aufl., Zürich, Schulthess Polygrapischer Verlag, 1998. (Gauch/Schluep/Emmenegger).

Gauch, Peter/Schluep, Walter R./ Schmid, Jörg/ Rey, Heinz: Schweizerisches Obligationenrecht Allgemeiner Teil: ohne ausservertragliches Haftpflichtrecht, B. II, 7.Aufl., Zürich, Schulthess Polygrapischer Verlag, 1998 (Gauch/Schluep/ Rey).

Gmür, Max: Kommentar zum Schweizerischen Zivilgesetzbuch: Obligationenrecht Art 68-183, B. VI, Bern, Verlag von Stämpfli Cie., 1917 (Becker).

Guhl, Theo/ Koller, Alfred/ Schnyder, Anton/ Druey, Jean Nicolas: Das Schweizerische Obligationenrecht: mit Einschluss des Handels- und Wertpapierrechts, 9. Aufl., Zürich, Schulthess, 2000. 
Haas, Hermann H./ Fuhlrott, Michael: "Ein Plädoyer für mehr Flexibilität bei Vertragsstrafen", NZA-RR, 2010/1, s.1-7.

Kahraman, Zafer: Karşılaştırmalı Hukukta Borcun Dış Üstlenilmesi (Borcun Nakli), İstanbul, Vedat Kitapçılık, 2013.

Keller, Max/ Schöbi, Christian: Allgemeine Lehren des Vertragsrechts: Das Schweizerische Schuldrecht, B. I, 3. Aufl., Basel/Frankfurt, Helbing \& Lichtenhahn, 1988.

Kocaağa, Köksal: Ceza Koşulu (Sözleşme Cezası), 2. Aufl. Ankara, Yetkin Yayınları, 2018.

Kohler, Josef: Lehrbuch des bürgerlichen Rechts, Berlin, Heymann, 1904.

Koller, Alfred: Schweizerisches Obligationenrecht Allgemeiner Teil: Handbuch des allgemeinen Schuldrechts ohne Deliktsrecht, 3. Aufl., Bern, Stämpfli Verlag AG, 2009.

Larenz, Karl: Allgemeiner Teil des Deutschen Bürgerlichen Recht, 6. Aufl., München, C.H. Beck Verlag, 1983.

Löwisch, Manfred/ Jagmann, Rainer/ Rieble, Volker: J. von Staudingers Kommentar zum Bürgerlichen Gesetzbuch: Buch 2: Recht der Schuldverhältnisse: §§ 328-345 (Vertrag zugunsten Dritter, Draufgabe, Vertragsstrafe), Berlin, Sellier-de Gruyter, 2015 (Staudinger/ Rieble).

Löwisch Manfred/ Rieble Volker/ Busche Jan/ Looschelders Dirk: J. von Staudingers Kommentar zum Bürgerlichen Gesetzbuch, Buch 2 - Recht der Schuldverhältnisse: $\S \S 397-$ 432 (Erlass, Abtretung, Schuldübernahme, Mehrheit von Schuldnern und Gläubigern), Berlin, Sellier-de Gruyter, 2017.

Niemann, Jan-Malte: "Vertragsbruch: Strafabreden in Formularbeitsverträgen”, RdA, 2013, Heft 2, s.92-101.

Nomer, Haluk N.: Borçlar Hukuku: Genel Hükümler, 16. Aufl., İstanbul, Beta, 2018.

Oertmann, Paul: Kommentar zum Bürgerlichen Gesetzbuche und seinen Nebengesetzen: Das Recht der Schuldverhältnisse, 2. Aufl., Berlin, Heymann, 1906.

Oğuzman, M. Kemal/ Öz, Turgut: Borçlar Hukuku: Genel Hükümler, B. II, 14. Aufl., İstanbul, Vedat Kitapçılık, 2018.

Oser, Hugo/Schönenberger, Wilhelm: Zürcher Kommentar zum Schweizerischen Zivilgesetzbuch, B. V: Erster Halbband, Obligationenrecht, Art 1-183 OR, 2. Aufl., Zürich, Schulthess, 1929.

Ozanemre Yayla, Tolunay: Alacağın Devri İşleminin Geçerliliği ve Sebeple Olan İlişkisi (İllîliği), Ankara, Turhan Kitabevi, 2019.

Özcan, Didem: Borcun Üstlenilmesi, İstanbul, On İki Levha Yayıncılık, 2017.

Palandt, Otto: Bürgerliches Gesetzbuch mit Nebengesetzen, B. VII, 76. Aufl., München, C.H. Beck Verlag, 2017 (Palandt/ Grüneberg).

Prütting, Hanns/ Wegen, Gerhard/ Weinreich, Gerd: Bürgerliches Gesetzbuch, Kommentar, 11. Aufl., Köln, Luchterhand Verlag, 2016.

Reck, Rudolf M.: Lohnrückbehalt, Kaution und Konventionalstrafe im schweizerischen Arbeitsrecht, Zürich, ADAG Administration \& Druck AG, 1983.

Richardi, Reinhard/ Wlotzke, Otfried/ Wissmann, Hellmut/ Oetker, Hartmut: Münchener Handbuch zum Arbeitsrecht: Individualarbeitsrecht, B. I, 3. Aufl., München, Verlag C.H. Beck, 2009.

Santoro, Dimitri: Die Konventionalstrafe im Arbeitsvertrag, Bern, Stämpfli Verlag AG, 2001.

Saymen, Ferit Hakkı: Borçlar Hukuku Dersleri, Umumi Hükümler, B.1, İstanbul, Akgün Matbaas1, 1950. 
Saymen, Ferit Hakkı / Elbir, Halid Kemal: Borçlar Hukuku Dersleri: Umumi Hükümler, B.1, İstanbul, İsmail Akgün Matbaas1, 1958.

Säcker, Franz Jürgen/ Rixecker, Roland/ Oetker, Hartmut/ Limperg, Bettina: Münchener Kommentar zum Bürgerlichen Gesetzbuch, B. II: Schuldrecht-Allgemeiner Teil, 7. Aufl., München, C. H. Beck, 2016 (MüKoBGB/ Gottwald, Art 339-345).

Schellhammer, Kurt: Schuldrecht nach Anspruchsgrundlagen: samt BGB Allgemeiner Teil, 9. Aufl., Heidelberg, C.F.Müller, 2014.

Schoch, Walter: Begriff, Anwendung und Sicherung der Konventionalstrafe nach schweizerischem Recht, Bern, Stämpfli \& Cie., 1935.

Söllner, Alfred: "Vertragsstrafen im Arbeitsrecht", AuR, 1981, s.97-105.

Stahel, Max: Die Konventionalstrafe mit spezieller Berücksichtigung des Schweizerischen Obligationenrechts, Zürich 1898.

Stürner, Rolf: Jauernig Bürgerliches Gesetzbuch: mit Rom I, Rom II, Rom III-VO, EG-UntVO/ HUntProt und EuErbVO, Kommentar, 16. Aufl., München, C.H.Beck, 2015 (Jauernig).

Tekinay, Selahattin Sulhi/ Akman, Sermet/ Burcuoğlu, Haluk/ Altop, Atilla: Tekinay Borçlar Hukuku: Genel Hükümler, 7. Aufl., İstanbul, Filiz Kitabevi, 1993.

Tunçomağ, Kenan: Türk Hukukunda Cezaî Şart, İstanbul, Baha Matbaası, 1963.

Tunçomağ, Kenan: Türk Borçlar Hukuku, Genel Hükümler, B.I, 6. Aufl., İstanbul, Sermet Matbaas1, 1976.

Von Tuhr, Andreas/ Escher, Arnold: Allgemeiner Teil des Schweizerischen Obligationenrechts, B. II, 3. Aufl., Zürich, Schulthess Polygraphischer Verlag AG, 1974. 